\title{
ON THE ATOMIC DECOMPOSITION OF $H^{1}$ AND INTERPOLATION
}

\author{
ROBERT SHARPLEY ${ }^{1}$
}

In [1] Coifman used the Fefferman-Stein theory of $H^{p}$ spaces [4] to decompose the functions of these spaces into basic building blocks (atoms), further clarifying their real variable nature. Coifman and Weiss have provided a comprehensive treatment of these ideas and many applications to harmonic analysis in [2]. In this note, we use the nontangential maximal function $N f$ to give an elementary proof of the decomposition of $H^{1}$ functions on the line and then characterize the Peetre $K$-functional for $H^{1}$ and $L^{\infty}$ in terms of $N f$.

Let $u$ be the harmonic extension [5] of $f$ to the upper half plane $\mathbf{R}_{+}^{2}$. For $x \in \mathbf{R}$, denote by $\Gamma_{2}=\left\{(z, y) \in \mathbf{R}_{+}^{2}:|x-z| \leq y\right\}$ the cone with vertex at $x$. The nontangential maximal function of $f$ is defined by $N f(x)=\sup \{|u(z, y)|:(z, y) \in$ $\left.\Gamma_{x}\right\}$. We define the (real) $H^{1}$ norm of $f$ to be the standard $H^{1}$ norm of $u+i v$, where $v$ is the harmonic conjugate of $u$ which satisfies $v(0)=0$. A classical result of Hardy and Littlewood asserts that $\|N f\|_{L^{1}} \leq c\|f\|_{H^{1}}$. For an interval $I$ an $H^{1}$-atom is any function $a_{I}$ such that $\int a_{I}=0$ and $\left|a_{I}\right| \leq|I|^{-1} \chi_{I}$ a.e.

PROPOSITION. ${ }^{2}$ Suppose $u$ is harmonic on an open square $S$ and continuous on $\bar{S}$. Then its average on $\partial S$ equals the average over the two diagonals.

PROOF. By dilating to a subcube of $S$ and then expanding back, we may assume that both $u$ and its harmonic conjugate $v$ are continuous on $\bar{S}$. Now $S$ is composed of four $15^{\circ}$ right triangles with common vertex the center of $S$. Let $T$ be the lower triangle and denote its edges by $L, B$ and $R$, where $B$ is the hypotenuse. Applying Cauchy's theorem to $u+i v$ on $T$ and taking real parts of the integrals gives

$$
0=\oint_{\partial T} u d x-v d y=\int_{B} u-\frac{1}{\sqrt{2}}\left(\int_{R+L} u+\int_{R} v-\int_{L} v\right) .
$$

Using rotations and symmetry, applying this argument to the three remaining subtriangles of $S$, and summing the resulting equations, we see that the terms involving $v$ cancel and we are left with our stated result.

THEOREM. If $N f \in L^{1}$, then we may write $f=\sum_{j} \lambda_{j} a_{j}$ so that the $a_{j}$ 's are atoms and the coefficients $\lambda_{j}$ satisfy

$$
\sum_{j}\left|\lambda_{j}\right| \leq 42\|N f\|_{L^{1}} .
$$

Received by the editors August 6, 1985.

1980 Mathematics Subject Classification. Primary 30D55, 42B25.

Key words and phrases. Hardy spaces, maximal function, interpolation.

${ }^{1}$ Research supported in part by NSF Grant MCS-8301360.

2 This result appears to be a part of the folklore. In fact, Jim Davis has mentioned to the author that it appeared on his qualifying exam at Stanford. In the mean time, Joel Shapiro has been able to provide an alternate proof using elementary potential theory. 
Proof. Since $u$ is continuous in $\mathbf{R}_{+}^{2}$, then $E_{k}=\left\{x: N f(x)>2^{k}\right\}$ is open in $\mathbf{R}$. Let $I(f)=\int_{I} f d x /|I|$ and define $F_{k}$ as the complement in $\mathbf{R}$ of $E_{k}$. We write $E_{k}$ as the disjoint union of its collection $C_{k}$ of open components and then decompose $f$ as a sum, $f=g_{k}+h_{k}$, where

$$
g_{k}=\sum_{I \in \mathcal{C}_{k}}[f-I(f)] \chi_{I}, \quad h_{k}=f \chi_{F_{k}}+\sum_{I \in \mathcal{C}_{k}} I(f) \chi_{I} .
$$

We claim that $\left|h_{k} Z\right| \leq 7 \times 2^{k}$ a.e. Clearly, the estimate holds on $F_{k}$ since $N f \leq 2^{k}$ there and $|f| \leq N f$ a.e. For the remaining set $E_{k}$, we fix an interval $I$ in $C_{k}$ and show that

$$
|I(f)| \leq 7 \times 2^{k} .
$$

Let $S_{\varepsilon}$ be the open square $I \times(\varepsilon,|I|+\varepsilon)$ in $\mathbf{R}_{+}^{2}$. By the Proposition and letting $\varepsilon \downarrow 0, I(f)$ is seen to equal four times the average of $u$ over the union of the two main diagonals less the sum of its averages over the three remaining sides. But the endpoints of $I$ belong to $F_{k}$, so the diagonals, sides and top of $S$ all belong to the "good" set for $u$, namely $\Gamma=\left\{(z, y) \in \Gamma_{x}: x \in F_{k}\right\}$. The definitions of $F_{k}$ and $N f$ imply that $u$ is bounded by $2^{k}$ on $\Gamma$ which establishes (3).

Following Coifman $[\mathbf{1}]$ and Herz $[\mathbf{6} \mathbf{f}]$, the atoms are defined by

$$
a_{I} \lambda_{I}^{-1}\left(g_{k}-g_{k+1}\right) \chi_{I}, \quad \lambda_{I}=21 \times 2^{k}|I|,
$$

for each $I \in C_{k}$ and all integers $k$. By telescoping and using both that $g_{k}-g_{k+1}=$ $h_{k+1}-h_{k}$ and that $g_{k+1}$ is supported in $E_{k+1} \subset E_{k}$, it follows that

$$
f=\sum_{k=\infty}^{\infty}\left(g_{k}-g_{k+1}\right)=\sum_{k} \sum_{I \in \mathcal{C}_{k}} \lambda_{I} a_{I} .
$$

Each $a_{I}$ is an atom since it is supported in $I$ and the estimate $\left\|a_{I}\right\|_{\infty} \leq|I|^{-1}$ follows from our $L^{\infty}$ estimate on the $h_{k}$,

$$
\left\|g_{k}-g_{k+1}\right\|_{\infty}=\left\|h_{k=1}-h_{k}\right\|_{\infty} \leq 7\left(2^{k+1}+2^{k}\right)=21 \times 2^{k} .
$$

To see that $a_{I}$ has mean value zero, it suffices to write it in the form

$$
a_{I}=\lambda_{I}^{-1}\left([f-I(f)] \chi_{I}-\sum_{\substack{J \in \mathcal{C}_{k+1} \\ J \subset I}}[f-J(f)] \chi_{J}\right) .
$$

To establish inequality (1) (subject to relabeling) we use

$$
\sum_{k} \sum_{I \in \mathcal{C}_{k}}\left|\lambda_{I}\right|+21 \sum_{k} 2^{k} \sum_{I \in \mathcal{C}_{k}}|I|=21 \sum_{k} 2^{k}\left|E_{k}\right|=21 \sum_{k}\left(2^{k+1}-2^{k}\right)\left|E_{k}\right| .
$$

Indeed by (5), summation by parts, and the fact $N f>2^{k}$ on $E_{k}$, we have

$$
\sum_{k} \sum_{I \in \mathcal{C}_{k}}\left|\lambda_{I}\right| \leq 42 \sum_{k} 2^{k}\left|E_{k} \backslash E_{k+1}\right| \leq 42 \int N f(x) d x .
$$

Fefferman, Rivière and Sagher $[\mathbf{3}]$ estimated the $K$-functional

$$
K(f, t)=\inf \left\{\|g\|_{H^{1}}+t\|h\|_{L^{\infty}}: g \in H^{1}, h \in L^{\infty}, f=g+h\right\}
$$

in terms of the "grand maximal" operator to describe interpolation spaces for the pair. We provide a description in terms of $N f$. Let $g^{*}$ denote the decreasing rearrangement of $|g|$. 
Corollary (OF THE PRoOF OF THE TheOREM). If $f$ belongs to $H^{1}+L^{\infty}$, then

$$
K(f, t) \sim \int_{0}^{t}(N f)^{*}(s) d s, \quad t>0 .
$$

ProOF. The subadditivity of the integral operator in (7) implies that it is dominated by $K(f, t)$. To establish the opposite estimate, we fix $t>0$ and select an integer $j$ so that $2^{j-1}<(N f)^{*}(t) \leq 2^{j}$. From the constructions in $(2)$, we see

$$
g_{j}=\sum_{k=j}^{\infty}\left(g_{k}-g_{k+1}\right)=\sum_{k=j}^{\infty}\left(\sum_{I \in C_{k}} \lambda_{I} a_{I}\right) .
$$

The estimate $\left\|h_{j}\right\|_{\infty} \leq 14(N f)^{*}(t)$ follows by our selection of the index $j$, while

$$
\left\|g_{j}\right\|_{H^{1}} \leq 42 c \int_{E_{j}} N f(x) d x \leq 42 c \int_{0}^{t}(N f)^{*}(s) d s
$$

is derived as in (5)-(6) using the identity (8). Combining these estimates completes the proof.

Minor modifications using $p$-atoms permit extension of these results to $H^{p}$ spaces $\left(\frac{1}{2}<p<1\right)$ on $\mathbf{R}$. Beginning with $N f$ and using classical techniques (theorems of Spanne-Stein and Hardy-Littlewood), these results provide a simplified approach to the various descriptions of $H^{p}(\mathbf{R})$ (duality, grand maximal operator). By conformally mapping the unit disc onto $\mathbf{R}_{+}^{2}$ and estimating the appropriate integrals obtained from the Proposition, one obtains the expected results for the circle. Exploiting a Fourier analytical technique of Calderón, Wilson has given a proof of the atomic decomposition into $L^{2}$ atoms for higher dimensions in [8], while the condition $N(u+i v) \in L^{1}$ is required in [7]. Finally, the author extends his thanks to Colin Bennett and Guido Weiss for valuable discussions related to this paper.

\section{REFERENCES}

1. R. R. Coifman, A real variable characterization of $H^{p}$, Studia Math. 51 (1974), 269-274.

2. R. R. Coifman and G. Weiss, Extensions of Hardy spaces and their uses in analysis, Bull. Amer. Math. Soc. 83 (1977), 569-645.

3. C. Fefferman, N. M. Rivière and Y. Sagher, Interpolation between $H^{p}$ space: The real method, Trans. Amer. Math. Soc. 191 (1974), 75-81.

4. C. Fefferman and E. M. Stein, $H^{p}$ spaces of several variables, Acta Math. 129 (1972), 137-193.

5. J. B. Garnett, Bounded analytic functions, Academic Press, New York, 1981.

6. C. Herz, $H^{p}$ spaces of martingales, $0<p \leq 1$, Z. Wahrsch. Verw. Gebiete 28 (1974), 189-205.

7. J. M. Wilson, $A$ simple proof of the atomic decomposition for $H^{p}\left(\mathbf{R}^{n}\right), 0<p \leq 1$, Studia Math. 74 (1982), 25-33.

8. __ On the atomic decomposition for Hardy spaces, Pacific J. Math. 116 (1985), 201207.

Department of MAThematics, University of South Carolina, Columbia, SOUTH CAROLINA 29208 